URLs

VAMP2

http://www.ncbi.nlm.nih.gov/ entrez/query.fcgi?db=gene\&c $\mathrm{md}=$ Retrieve\&dopt=full report\&list_uids $=22318$

\title{
SYNAPTIC PHYSIOLOGY
}

\section{More than a flicker}

A new study from Richard Tsien's laboratory provides compelling evidence that 'kiss-and-run' is the main type of vesicle fusion at hippocampal synapses. The kinetics of kiss-andrun and vesicle re-use vary strikingly with stimulus frequency, which might allow small nerve terminals to conserve limited vesicular resources in the face of a demanding array of input patterns.

Of the many vesicles present at small central synapses, only $\sim 30$ are thought to be actively involved in transmitter release. Classically, fusion with the plasma membrane leads to the full collapse of each vesicle, involving complete aqueous continuity between the vesicle lumen and the extracellular solution. Retrieving, refilling and repriming a collapsed vesicle before the next release event could take several tens of seconds - an interval that would restrict the speed of synaptic information transfer.

Evidence gathered during the past few years for a second, nonclassical mode of fusion, known as kiss-and-run or 'flicker', seemed to provide a solution to the problem. In the case of kiss-and-run, vesicles fuse transiently with the plasma membrane to release their contents, but remain intact for refilling and further transmitter release (re-use). However, because vesicle fusion at small terminals is particularly difficult to study, the defining features of kiss-and-run have remained unclear, and its existence has even been called into question.

Tsien and colleagues developed a novel fluorescence quenching technique to overcome some of the limitations of methods used previously to record or visualize kiss-andrun fusion events. They used a small hydrophilic quencher (bromophenol blue) that could rapidly enter vesicles from the extracellular medium during brief fusion events to quench FM dye or enhanced green fluorescent protein (EGFP) that was tagged to the lumenal domain of the vesicular protein VAMP2 (synaptobrevin 2).

They confirmed that during first fusion, FM1-43 was partially trapped in vesicles loaded with the dye - firm evidence for rapid vesicle retrieval characteristic of kiss-and-run, rather than classical collapse - and that the prevalence of kiss-and-run was strongly frequency dependent, increasing at lower frequencies to $\sim 80 \%$. What's more, the researchers were able to probe the kinetics of first fusion and the rate of vesicle re-use, and to show that these measures were also influenced by stimulation frequency.

Tsien and colleagues have added considerably to our understanding of the characteristics of kiss-and-run, and have provided a powerful technique to answer the many questions that remain about the physiological role of this mode of fusion.

Rebecca Craven

ORIGINAL RESEARCH PAPER Harata, N. C. et al. Frequency-dependent kinetics and prevalence of kiss-and-run and reuse at hippocampal synapses studied with novel quenching methods. Neuron 49, 243-256 (2006)

FURTHER READING Harata, N. et al. Limited numbers of recycling vesicles in small CNS nerve terminals: implications for neural signaling and vesicular cycling. Trends Neurosci. 24, 637-643 (2001) |Rizzoli, S. O. \& Betz, W. J. Synaptic vesicle pools. Nature Rev. Neurosci. 6, 57-69 (2005) WEB SITE

Tsien's laboratory: http://www.stanford.edu/ group/Tsienlab/ 